\title{
Determination of Estazolam in Plasma by High-Performance Liquid Chromatography with Solid-Phase Extraction
}

\author{
Masatomo Miura,* Tadashi OhKubo, ${ }^{* \dagger}$ Kazunobu Sugawara,* Naoyuki OKuyama,** and \\ Koichi OTANI**
}

*Department of Pharmacy, Hirosaki University Hospital, Hon-cho, Hirosaki 036-8563, Japan

**Department of Neuropsychiatry, Yamagata University Hospital, Yamagata 990-9585, Japan

\begin{abstract}
A high-performance liquid chromatography (HPLC) assay was developed for the determination of estazolam in human plasma. Estazolam and alprazolam as an internal standard were detected by ultraviolet absorbance at $240 \mathrm{~nm}$. Estazolam in plasma was extracted by a rapid and simple procedure based on cyanopropyl bonded-phase extraction. Chromatographic separation was achieved with a reversed-phase $\mathrm{C}_{8}-5$ column using a mobile phase of $0.5 \%$ potassium dihydrogenphosphate $(\mathrm{pH} 4.5)$-acetonitrile $(70: 30, \mathrm{v} / \mathrm{v})$. The determination of estazolam was possible in the concentration range of $1.0-200.0 \mathrm{ng} / \mathrm{mL}$. The mean recovery of estazolam added to plasma was $96.1 \pm 1.5 \%$ with coefficients of variation of less than $5.5 \%$. This method is applicable for accurately monitoring the plasma level of estazolam in healthy subjects participating in scientific research.
\end{abstract}

(Received February 4, 2002; Accepted March 18, 2002)

\section{Introduction}

Estazolam (8-chloro-6-phenyl-4H-S-triazolo-4,3- $\alpha$-1,4-benzodiazepine) is a triazolobenzodiazepine derivative (Fig. 1a), mainly prescribed as a sedative and hypnotic agent. ${ }^{1}$ It is classified as a medium-acting benzodiazepine. ${ }^{2}$ Estazolam generates 4hydroxyestazolam, its main metabolite, which may retain some pharmacological activity, and 1-oxo-estazolam in humans. ${ }^{3}$ However, these metabolites are unlikely to contribute significantly to the overall pharmacological activity because of their negligible concentration and rapid glucuronidation. ${ }^{3}$ Estazolam is also quickly absorbed in humans when administered orally. The plasma half-life has been reported to be approximately $17 \mathrm{~h}$, and does not appear to induce a drug metabolism enzyme. ${ }^{4}$ However, the effects of cytochome P-450 isozyme (CYP) on estazolam metabolism have not yet been clarified. Although a potential drug interaction of estazolam with ritonavir has been a warning in the package insert of estazolam products, ${ }^{5}$ there have been no clinical reports about drug interactions involving estazolam. Therefore, a study of drug interactions involving estazolam should be performed from the point of view of drug safety.

The measurement methods of estazolam by GC, 4,6,7 GC-MS and HPLC $^{9,10}$ were described in previous papers. However, several extraction methods of estazolam with liquid-liquid extraction were used for plasma sample preparation. These methods were not satisfactory because they were too tedious and time consuming. We have reported a simple extraction method of several drugs by using a solid-phase extraction cartridge. ${ }^{11-13}$ On the other hand, a C18 stationary phase was used for an analytical column in all previous HPLC analytical methods for estazolam. ${ }^{8-10}$ However, we think that the $\mathrm{C} 18$

$\doteqdot$ To whom correspondence should be addressed. analytical column is not always suitable for the analysis of estazolam and several drugs because of the analytical time required and lack of separation efficiency. We recently reported on HPLC analytical methods for several drugs by using a C8 analytical column. ${ }^{14-16}$ More efficient separation and a shorter analytical time were obtained in the C8 HPLC column than in the $\mathrm{C} 18$ column, depending on the properties of the analytical drugs. We have appreciated the benefit of the $\mathrm{C} 8$ column in drug analysis by HPLC. ${ }^{14-16}$ In the present paper, we describe a rapid and simple solid-phase extraction method and selective HPLC method for the determination of estazolam levels in human plasma. The method was applied to studies of pharmacokinetics of estazolam in healthy volunteers.

\section{Experimental}

\section{Reagents and chemicals}

Estazolam was donated and estazolam tablets ( $2 \mathrm{mg}$ tablet of EURODIN) were purchased from Takeda Pharmaceutical Co., Ltd., (Osaka, Japan). Alprazolam was kindly donated by the Upjohn Company (Kalamazoo, MI, USA). A Sep-Pak CN cartridge was purchased from Waters Co. (Bedford, MA, USA). a)

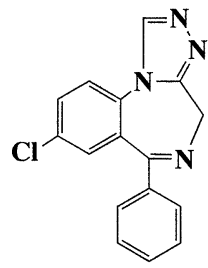

b)<smiles>Cc1nnc2n1-c1ccc(Cl)cc1C(c1ccccc1)=NC2</smiles>

Fig. 1 Chemical structures of (a) estazolam and (b) alprazolam 
Table 1 Retention times for each benzodiazepine derivatives under the same chromatographic condition

\begin{tabular}{lc}
\hline Compound & Retention time/min \\
\hline Estazolam & 14.3 \\
Alprazolam & 18.0 \\
Triazolam & 19.3 \\
Nitrazepam & 16.4 \\
Flurazepam & 8.0 \\
Flunitrazepam & 20.3 \\
Oxazepam1 & 3.0 \\
Clonazepam & 21.3 \\
Bromazepam & 24.3 \\
\hline
\end{tabular}

All solvents used were of HPLC grade (Wako Pure Chemical Industries, Osaka, Japan). All other reagents and chemicals were purchased from Wako Chemical Industries or Nacalai Tesque (Kyoto, Japan).

\section{Apparatus}

The apparatus used for HPLC was a Model PU-980 chromatography pump (JASCO, Tokyo, Japan) equipped with a JASCO UV-970 ultraviolet detector. The wavelength was set at $240 \mathrm{~nm}$. Test samples were introduced using a JASCO AS1555-10 interigent auto sampler with an effective volume of $100 \mu \mathrm{L}$. The HPLC column used was a Develosil $\mathrm{C}_{8}-5$ stationary phase ( $5 \mu \mathrm{m}$, Nomura Chemical, Aichi, Japan). A stainless-steel analytical column $(150 \times 4.6 \mathrm{~mm}$ i.d. $)$ was packed in our laboratories by a conventional high-pressure slurry-packing procedure. The mobile phase consisted of $0.5 \%$ $\mathrm{KH}_{2} \mathrm{PO}_{4}(\mathrm{pH} 4.5)$-acetonitrile (70:30, v/v), which was degassed in an ultrasonic bath prior to use. A flow-rate of $1.0 \mathrm{~mL} / \mathrm{min}$ was used at ambient temperature. Before mixing, the $\mathrm{pH}$ of the $0.5 \% \mathrm{KH}_{2} \mathrm{PO}_{4}$ was adjusted with $50 \%$ phosphoric acid, and it was degassed ultrasonically.

\section{Extraction method}

After alprazolam $(50 \mathrm{ng})$ in methanol $(10 \mu \mathrm{L})$ was added to plasma samples $(1 \mathrm{~mL})$ as an internal standard, the plasma samples were diluted with $5 \mathrm{~mL}$ of $1 \mathrm{M}$ sodium chloride, and the solution was briefly mixed. The mixture was applied to a Sep-Pak CN cartridge that had previously been activated with $5.0 \mathrm{~mL}$ acetonitrile and water. The cartridge was then washed with $10 \mathrm{~mL}$ of water. The desired fraction was eluted with 5 $\mathrm{mL}$ of $20 \%$ acetonitrile in water. The eluate was evaporated to dryness in a vacuum at $60^{\circ} \mathrm{C}$ by a rotary evaporator (Iwaki, Tokyo, Japan). The residue was dissolved in $100 \mu \mathrm{L}$ methanol. The samples were injected into the HPLC apparatus.

\section{Calibration graphs}

Known amounts of estazolam in the range of $1.0-200 \mathrm{ng} / \mathrm{mL}$ were added to blank plasma samples. The plasma samples were treated according to the determination procedure described above. Graphs were constructed of the peak-height ratio of estazolam to alprazolam as an internal standard, and plotted against the concentration of estazolam.

\section{Recovery and assay validation}

The spiked samples were prepared by adding 1.0, 10.0, 25.0, 50.0, 100.0, and $200.0 \mathrm{ng} / \mathrm{mL}$ estazolam to blank plasma. Sample extraction and the subsequent HPLC technique were carried out as described above. The control samples were not extracted, but were directly evaporated and the residues were reconstituted in methanol. An external standard (instead of the
Table 2 Accuracy and precision of the determination of estazolam in human plasma $(n=6)$

\begin{tabular}{lcccc}
\hline \multirow{2}{*}{$\begin{array}{c}\text { Added/ } \\
\text { ng mL }\end{array}$} & \multirow{2}{*}{$\begin{array}{c}\text { Found/ng mL } \\
\text { (mean } \pm \text { S.D. }\end{array}$} & \multirow{2}{*}{$\begin{array}{c}\text { Recovery, } \\
\%\end{array}$} & & \multicolumn{2}{c}{ C.V., \% } \\
\cline { 4 - 5 } & & & Inter-day & Intra-day \\
\hline 1.0 & $0.93 \pm 0.04$ & 93.4 & 4.7 & 4.6 \\
10.0 & $9.60 \pm 0.47$ & 96.0 & 4.9 & 4.4 \\
25.0 & $24.38 \pm 0.73$ & 97.5 & 3.0 & 2.9 \\
50.0 & $48.71 \pm 2.25$ & 97.4 & 4.6 & 4.2 \\
100.0 & $95.90 \pm 5.32$ & 95.9 & 5.5 & 5.3 \\
200.0 & $193.17 \pm 5.39$ & 96.6 & 2.9 & 3.1 \\
\hline
\end{tabular}

internal one) was added before ample evaporation. The recoveries were determined by a comparison between the solidphase extraction and a non-extraction control. The validation method was determined by calibration and recovery experiments. The recovery was calculated as the percentage of the nominal concentration: recovery $=$ [observed concentration/nominal concentration] $\times 100$. The between-run coefficient of variation was calculated from measurements on six separate days. The within-run coefficient of the variation was derived from an analysis of six quality-control samples at each concentration on the same day. The calibration curves were required to have a correlation value of at least 0.9992 . The detection limit was defined as the quantity of the sample after preparation and extraction which had a coefficient of variation of less than $20 \%$ (signal-to-noise ratio $=5.0$ ).

\section{Application to pharmacokinetics studies}

The method was used to quantitate the estazolam plasma concentration in a single-dose pharmacokinetic study. The subjects were three healthy male volunteers. This study was approved by the Ethics Committee of Yamagata University Hospital, and all subjects gave written, informed consent. Three healthy male volunteers took $4 \mathrm{mg}$ of the EURODIN brand of estazolam (Takeda) in a fasting state. Blood samples $(10 \mathrm{~mL})$ were collected by vein puncture at $0.5,1,2,3,4,6,8,10,12$, $24,36,48,60$ and $72 \mathrm{~h}$ after oral administration. The plasma was separated by centrifugation at $1900 \mathrm{~g}$ for $15 \mathrm{~min}$ and stored at $-30^{\circ} \mathrm{C}$ until an analysis. A pharmacokinetic analysis of estazolam was carried out by the following methods. The total area under the observed plasma concentration-time curve (AUC) was calculated by using the linear trapezoidal rule. The terminal elimination half-life was obtained directly from the two-compartment model. The peak plasma level $\left(C_{\max }\right)$ and time to reach the peak $\left(t_{\max }\right)$ were obtained directly from the profile.

\section{Results}

Under the described chromatographic condition, estazolam and alprazolam as an internal standard were separated. The retention times for estazolam, alprazolam, triazolam, nitrazepam, flurazepam, flunitrazepam, oxazepam, clonazepam and bromazepam were 14.3, 18.0, 19.3, 16.4, 8.0, 20.3, 13.0, 21.3 and $24.3 \mathrm{~min}$ (Table 1). No other benzodiazepine derivatives were piled up on the chromatographic peaks of estazolam and alprazolam. Typical chromatograms of drug-free plasma and plasma spiked with alprazolam from estazolamtreated volunteers are shown in Fig. 2. The separation of estazolam and alprazolam was satisfactorily obtained, and the isolation of interfering peaks in biological matrix from the 

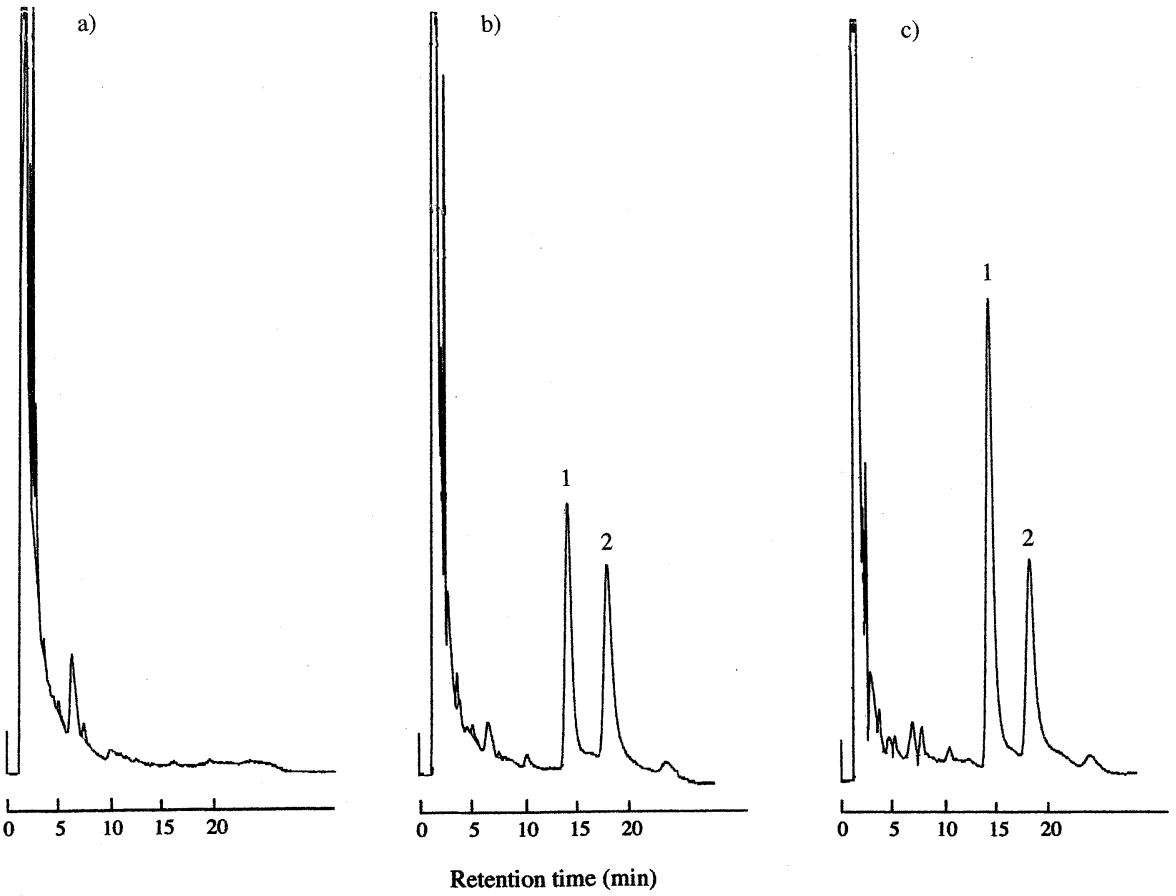

Fig. 2 Typical chromatograms of a) plasma blank, b) spiked with estazolam $(50 \mathrm{ng} / \mathrm{mL})$ and alprazolam (I. S.) in plasma and c) volunteer plasma sample. 1, estazolam; 2, alprazolam (I. S.).

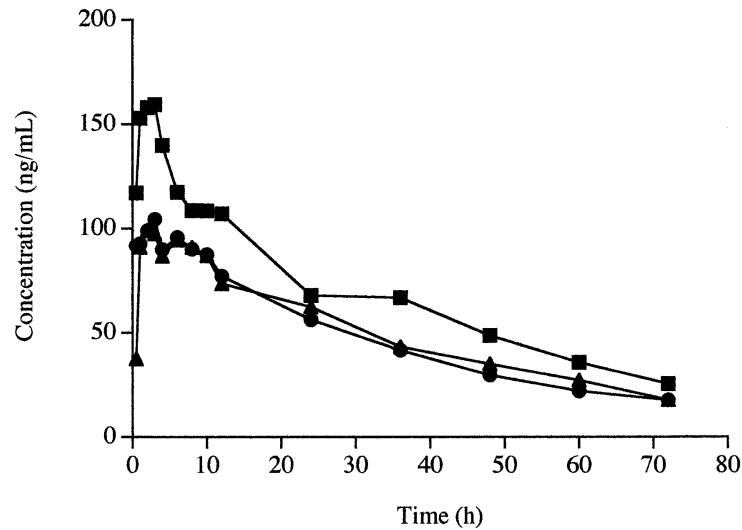

Fig. 3 Plasma concentration-time profile of estazolam after a single $4 \mathrm{mg}$ oral dose of estazolam to three healthy volunteers.

analyzed peaks was then established by our extraction method and chromatographic system. The calibration curves for estazolam in plasma were linear in the concentration range of $1.0-200 \mathrm{ng} / \mathrm{mL}$. The limit of detection for estazolam was 0.5 $\mathrm{ng} / \mathrm{mL}$.

The results of recovery studies are given in Table 2. The recovery of estazolam was determined by adding six known concentrations $(1.0,10,25,50,100$ and $200 \mathrm{ng} / \mathrm{mL})$ to drugfree plasma. The recovery values for estazolam were 93.4 $97.5 \%$ in plasma in the concentration range was $1.0-200$ $\mathrm{ng} / \mathrm{mL}$. The within-run and between-run assay variations for estazolam were less than $5.5 \%$ in plasma. The accuracy and precision of the proposed method were defined from these results.

The concentrations of estazolam in plasma samples from estazolam-treated volunteers were determined using our present method. The time course of the concentration of estazolam in plasma samples from three healthy volunteers receiving $4 \mathrm{mg}$
Table 3 Pharmacokinetic parameters of estazolam after a single oral $4 \mathrm{mg}$ dose of estazolam in three healthy volunteers

\begin{tabular}{crrrccc}
\hline \multirow{2}{*}{ Subject } & \multicolumn{6}{c}{ Parameter } \\
\cline { 2 - 7 } & $\begin{array}{c}C_{\max } / \\
\mathrm{ng} \mathrm{mL}\end{array}$ & $\begin{array}{c}t_{\max } / \\
\mathrm{h}\end{array}$ & $\begin{array}{c}t_{1 / 2} / \\
\mathrm{h}\end{array}$ & $\begin{array}{c}\mathrm{AUC}_{(0-72)} / \\
\mathrm{ng} \mathrm{h} \mathrm{mL}^{-1}\end{array}$ & $\begin{array}{c}\mathrm{AUC}_{(0-\infty)} / \\
\mathrm{ng} \mathrm{h} \mathrm{mL}^{-1}\end{array}$ & $\begin{array}{c}\mathrm{Cl}_{\mathrm{t}} / \\
\mathrm{L} \mathrm{h}^{-1}\end{array}$ \\
\hline 1 & 104.4 & 3 & 26.6 & 3439.6 & 4074.0 & 1.0 \\
2 & 99.1 & 2 & 28.8 & 3592.0 & 4380.2 & 0.9 \\
3 & 159.5 & 3 & 26.0 & 4902.3 & 5862.6 & 0.7 \\
\hline
\end{tabular}

estazolam orally was determined using the proposed method (Fig. 3). The pharmacokinetic parameters of three healthy volunteers are given in Table 3. The time required to reach the maximum concentration of estazolam was $2-3 \mathrm{~h}$, and the concentration was $104.4-159.5 \mathrm{ng} / \mathrm{mL}$. The half-life values of the $\beta$-phase were $26.0-28.8 \mathrm{~h}$. The $\mathrm{AUC}_{(0-72)}$ and $\mathrm{AUC}_{(0-\infty)}$ values were 3439.6 - $4902.3 \mathrm{ng} \mathrm{h} / \mathrm{mL}$ and 4074.0 - $5862.6 \mathrm{ng}$ $\mathrm{h} / \mathrm{mL}$.

\section{Discussion}

The method presented here is rapid, specific and sensitive enough to allow the quantitation of estazolam in the plasma of volunteers being treated with estazolam. Initially, our efforts were directed towards developing an efficient chromatographic system for the mutual separation of benzodiazepine derivatives and a more efficient analytical time than the previous HPLC method of estazolam. In previous studies, an octadecyl column was used for estazolam HPLC analytical methods. ${ }^{8-10}$ However, we have shown that the $\mathrm{C} 8$ analytical column, which provides a more efficient resolution and a shorter analytical time than the C18 phase, has a greater efficacy for the analysis of several drugs by the HPLC method. ${ }^{14-16}$ Therefore, the C 8 analytical column was used for our present HPLC analytical method in plasma estazolam. The retention times for estazolam and 
alprazolam were 14.3 and $18.0 \mathrm{~min}$, because sharper pecks were obtained by using the $\mathrm{C} 8$ analytical column. The peaks of estazolam and alprazolam on the chromatogram were not interfered with by other benzodiazepine derivatives.

The extraction method of estazolam and alprazolam (I.S.) in plasma was established using a Sep-Pak CN cartridge and $1 \mathrm{M}$ $\mathrm{NaCl}$ as an applied solution. Low interference from endogenous components of plasma was obtained by this method. Although previous determination methods for estazolam in plasma by HPLC were essentially performed by quantitative liquid-liquid extraction, ${ }^{8-10}$ such procedures are tedious and time-consuming. In previous papers, we have described a simple solid-phase extraction method for various drugs. ${ }^{11-13}$ Therefore, we established a simple solid-phase extraction method for estazolam and alprazolam as an internal standard in plasma using a Sep-Pak CN cartridge. High ionic 1 $\mathrm{M} \mathrm{NaCl}$ was used for the absorption of plasma estazolam and alprazolam as an internal standard on Sep-Pak CN stationary phases, which were achieved at low interference from endogenous components of plasma.

The sensitivity and the calibration range of the present method were appropriate for the therapeutic drug monitoring of estazolam in healthy volunteers. In previous papers, we described determinations of estazolam in plasma by the HPLCUV detection method. ${ }^{8,9}$ However, the detection limit of estazolam was not satisfactory for monitoring of the late period after drug dosing in a volunteer study. We therefore established a highly sensitive $(1 \mathrm{ng} / \mathrm{mL})$ HPLC method using Sep-Pak CN solid-phase extraction. The precision of the data in the present study indicates that this assay has acceptable reproducibility. The correlation coefficients for standard calibration curves were consistently greater $(r=0.9992)$. Therefore, the present HPLC method could be applicable for monitoring of low concentrations of estazolam in plasma.

Pharmacokinetic parameters of estazolam have been reported in previous papers., ${ }^{4,17}$ The times required to reach the maximum concentration, and the half-life values of estazolam in the present study were similar when compared with previous data. 4,7,17 The isozyme of cytochrome P-450 (CYP) metabolized estazolam has not been declared in any previous study, and the drug interactions of estazolam have not been shown in previous reports. Therefore, an in vitro metabolic study of estazolam should be conducted using several recombinant CYP isozymes, and the pharmacokinetic analysis of drug interaction with estazolam will require further study. The results of the present study indicate that this HPLC method is a sensitive and precise means for measuring low levels of estazolam in plasma. We are now planning to apply this sensitive method for further metabolic and pharmacokinetic studies.

\section{References}

1. M. W. Pierce and V. S. Shu, Am. J. Med., 1990, 88 (supplement), 3A 6s.

2. D. J. Greenblatt, L. G. Miller, and R. I. Shader, Am. J. Med., 1990, 88 (supplement), 3A 18s.

3. J. M. Machinist, B. A. Bopp, D. J. Anderson, G. R. Granneman, R. C. Sonders, K. Tolman, K. Buchi, and D. Rollins, Xenobiotica, 1986, 16, 11.

4. M. D. Allen, D. J. Greenblatt, and J. D. Arnold, Psychopharmacol., 1979, 66, 267.

5. EURODIN insert package, Takeda Pharmaceutical Co., Ltd., Osaka, Japan, 1998.

6. J. F. Kell and D. J. Greenblatt, J. Chromatogr., 1993, 621, 102.

7. L. Dal Bo, F. Verga, A. Marzo, P. La Commare, and M. L. DeVito, Pharmacol. Res., 1997, 35, 329.

8. H. Inoue, Y. Maeno, M. Iwasa, J. Monma, and R. Matoba, J. Chromatogr., 1997, 701, 47.

9. A. S. di Tella, P. Ricci, C. Di Nunzio, and P. Cassandro, J. Anal. Toxicol., 1986, 10, 65.

10. A. Boukhabza, A. A. J. Lugnier, P. Kintz, and P. Mangin, J. Anal. Toxicol., 1991, 15, 319.

11. T. Ohkubo, R. Shimoyama, and K. Sugawara, J. Pharm. Sci., 1992, 81, 947.

12. T. Ohkubo, T. Osanai, K. Sugawara, M. Ishida, K. Otani, K. Mihara, and N. Yasui, J. Pharm. Pharmacol., 1994, 47, 340.

13. T. Nagasaki, T. Ohkubo, K. Sugawara, N. Yasui, H. Furukori, and S. Kaneko, J. Pharm. Biomed. Anal., 1999, 19, 595.

14. T. Nagasaki, T. Ohkubo, K. Sugawara, N. Yasui, K. Otani, and S. Kaneko, J. Chromatogr. B, 1998, 714, 293.

15. R. Shimoyama, T. Ohkubo, and K. Sugawara, Ann. Clin. Biochem., 2000, 37, 210.

16. R. Shimoyama, T. Ohkubo, and K. Sugawara, J. Liq. Chrom. Rel. Technol., 2000, 23, 587.

17. L. E. Gustavson and P. J. Carrigan, Am. J. Med., 1990, 88 (supplement), 3A 2s. 